\title{
SECTOR EXTERIOR Y CRECIMIENTO EN LA ESPAÑA AUTÁRQUICA
}

\author{
ELENA MARTÍNEZ RUIZ * \\ Universidad Carlos III de Madrid
}

\section{RESUMEN}

En el presente trabajo se presentan nuevos datos de balanza de pagos. La nueva evidencia sugiere que la restricción a la importación se relajó en cierta medida durante el primer lustro de los cincuenta, gracias a los aumentos en las entradas de divisas. Este incremento se basó en el impulso que las ventas de bienes y servicios experimentaron a partir de 1949. A continuación se analizan los factores explicativos del aumento de las ventas españolas. Así se estudian el efecto de un hipotético cambio en la política comercial y la influencia del contexto internacional.

\section{ABSTRACT}

This paper analyses new balance of payment data for Spain in the autarkic period. New evidence suggests that import restrictions where relaxed to a certain extent during the first half of the $1950 \mathrm{~s}$ thanks to an increase of the entry of foreign currency. This increase was due to the rise of exports of goods and services beginning in 1949. The paper studies several explanatory factors for this increase. In particular the effect of a hypothetical change in commercial policy and the influence of the international context are considered.

\section{INTRODUCCIÓN}

La limitada capacidad para importar de la economía española se señala frecuentemente como el principal obstáculo al crecimiento de la economía

* La autora agradece los comentarios de los asistentes al seminario «Nuevas interpretaciones en la Historia Económica de España» celebrado en Cuenca los día 29 y 30 de junio de 2000, en especial de Pablo Martín Aceña y José María Serrano Sanz, así como las observaciones de dos evaluadores anónimos. María Jesús Asensio, Regina Grafe, Alfonso Herranz y Antonio Tena hicieron también valiosas observaciones. Todos los errores son responsabilidad mía. 
española durante el período autárquico. La escasez de materias primas y bienes intermedios perjudicó el normal desarrollo de las actividades productivas, mientras la insuficiente importación de bienes de equipo condenó a la economía española a trabajar con tecnologías obsoletas y poco eficientes. La política comercial y cambiaria del régimen franquista fue la principal responsable de tal restricción. El exacerbado proteccionismo, que envolvió a la economía española en una maraña de licencias previas, cuotas de importación y cambios múltiples, y la errónea elección de la paridad de la peseta, muy por encima de su valor real, son los más significativos ejemplos de la nefasta e intensa intervención estatal, causante de los deficientes resultados obtenidos por la economía española en estos años.

Sin embargo, la economía española comenzó a crecer desde los primeros años cincuenta. Tras doce años de lentísima recuperación, en 1951 se superó finalmente el nivel de producción anterior a la Guerra Civil y a partir de ese momento la economía española salió de su estancamiento, aunque siguió creciendo a un ritmo inferior al de otros países europeos ${ }^{1}$. Este artículo examina hasta qué punto el sector exterior contribuyó al proceso de crecimiento de estos años. Con este objetivo se presentan, en primer lugar, nuevos datos de balanzas de pagos que reflejan un cambio en la evolución de las relaciones económicas exteriores de España a partir de 1949. Los rasgos más llamativos de este cambio son, por un lado, el apreciable, aunque efimero, crecimiento de las ventas de bienes y servicios y la pérdida de importancia de las entradas de capital a partir de la ruptura del Protocolo Franco-Perón, y, por otro, el progresivo aumento de las compras exteriores, sobre todo de materias primas, bienes intermedios y maquinaria. Esta parcial relajación del cuello de botella de las importaciones contribuyó en cierta medida al proceso de crecimiento económico que se puso en marcha en estos años.

Por tanto, resulta de interés indagar en los elementos explicativos del cambio en el desarrollo de las relaciones económicas exteriores de los primeros años cincuenta. El primer factor estudiado es el impacto de la política comercial y cambiaria a partir de 1948 . Más concretamente, se analiza si hubo una modificación en el carácter general de la política económica exterior y si éste puede ser interpretado como un paso hacia la liberalización. Tras responder negativamente a ambas cuestiones, se estudia la influencia del cambiante contexto político y económico en Europa sobre el comercio español. La creciente demanda de las economías europeas en auge, por

\footnotetext{
'Prados de la Escosura y Sanz (1996).
} 
un lado, y la definitiva apertura de los mercados internacionales tras el fin del bloqueo, por otro, ofrecieron un marco muy favorable para el desarrollo de las relaciones económicas con el exterior. Sin embargo, el propicio entorno sirvió para mantener un sistema que ya había dado síntomas de agotamiento y la economía española sólo aprovechó parcialmente las oportunidades que la situación internacional le brindaba. En este sentido, puede afirmarse que se dejó pasar una inmejorable ocasión para la liberalización de la economía española, que aún hubo de esperar diez años más.

\section{EL SECTOR EXTERIOR TRAS LA SEGUNDA GUERRA MUNDIAL}

A pesar de las evidentes continuidades, en la evolución del sector exterior autárquico se pueden distinguir varias etapas ${ }^{2}$. Una primera etapa abarcó los años de la Segunda Guerra Mundial y estuvo marcada por las excepcionales circunstancias impuestas por el conflicto. En España, la guerra causó un estancamiento de la actividad comercial, fundamentalmente en cuanto a importación, así como un notable aumento de los servicios prestados al extranjero, en especial fletes. Los superávit en la balanza por cuenta corriente fueron utilizados para la reconstrucción de las reservas de oro consumidas durante la Guerra Civil ${ }^{3}$. La segunda etapa se extendió hasta 1954 y, en ella, lo más destacable es el mantenimiento de una cierta estabilidad, obtenida a base de limitar los gastos en función de los ingresos. La balanza básica española se mantuvo en una situación cercana al equilibrio entre 1946 y 1954. Sin embargo, este frágil equilibrio tuvo rasgos muy diferentes antes y después de 1949. Para analizarlos es preciso fijar la atención en la situación de la balanza por cuenta corriente, esto es, a la evolución de la posición deudora o acreedora de la economía española frente al exterior o, expresado en otros términos, a su capacidad para generar ahorro suficiente para financiar la inversión y el gasto público. Durante los cuatro primeros años (1946-1949), la balanza por cuenta corriente arrojó un déficit permanente, sólo compensado por el superávit de la balanza de capital a largo plazo, gracias a los préstamos argentinos. Por el contrario, entre 1950 y 1954, pequeños déficit en la balanza por cuenta corriente eran compensados por los ligeros superávit en la balanza de capital. Los

${ }^{2} \mathrm{El}$ análisis que aquí se presenta está basado en la última reconstrucción de las balanzas de pago del período autárquico, recogidas en Martínez Ruiz (1998).

${ }^{3}$ Véase Martín Aceña et al. (1998). 
responsables de esta situación fueron, por un lado, la exportación, que experimentó un fuerte aumento en estos años, y, por otro, los préstamos de bancos privados estadounidenses, que tomaron el relevo de las líneas de crédito argentinas, permitiendo un saldo positivo también en la balanza de capital. En 1954, la ayuda americana permitió que las operaciones corrientes abandonasen los números rojos. Fue el último año de estabilidad. A partir de 1955 comenzó una nueva fase que terminó con la preparación del Plan de Estabilización. Durante estos años, el progresivo empeoramiento por cuenta corriente planteó serios problemas, dada la escasa capacidad para atraer capital exterior. Ésta es, en mi opinión, la característica más destacada de la segunda mitad de los cincuenta. A pesar de la ayuda americana, que permitió un ligero repunte de los invisibles y las transferencias en 1954 y 1955, el creciente déficit de la balanza comercial, provocado por el aumento de las importaciones, arrastró a la economía española a una situación insostenible. Enfrentados a una insuficiente financiación exterior y agotadas las reservas por las ventas de oro en 1957 y 1958, la reforma se haría inevitable. El paulatino empeoramiento del desequilibrio exterior constituyó, sin duda, un aspecto fundamental de la crisis del final de decenio y, sin embargo, es posible que el despegue del crecimiento económico en España encontrara cierto apoyo en el desarrollo de las relaciones internacionales en el primer lustro de los cincuenta. Merece la pena, por tanto, indagar algo más en la evolución de las diferentes partidas de la balanza de pagos española entre 1948 y 1954, para identificar esos síntomas de dinamismo.

La evolución del comercio exterior durante el período autárquico está recogida en el Gráfico 1. La balanza comercial sufrió, a partir de 1946, un progresivo empeoramiento causado principalmente por el aumento de la importación, que creció a un ritmo superior al de la exportación hasta 1948. Solamente la existencia de significativos mecanismos de bilateralización del comercio permitió este pequeño auge importador. Entre ellos, fueron de crucial importancia los acuerdos firmados con Argentina, base de buena parte de la importación realizada durante estos tres años. Entre 1946 y 1949, la distribución espacial del comercio español varió notablemente. Descartados los tradicionales socios europeos, bien por el bloqueo, bien por los problemas de la reconstrucción, España hubo de hallar fuentes alternativas de aprovisionamiento. Los países del continente americano, sobre todo Argentina y EE UU, se presentaron, no como la opción más ventajosa, sino como la única posible. Estados Unidos fue durante años el único país capaz de suministrar mercancías imprescindibles para un nor- 
mal desenvolvimiento de la economía. En este sentido, España no se diferenció del resto de países de Europa. Por el contrario, las compras a Argentina, realizadas en el especial marco que constituyeron los acuerdos entre ambos gobiernos suponen una particularidad española. Los créditos a largo plazo y, sobre todo, los comerciales concedidos por el país sudamericano evitaron la total paralización de la importación española. Pero no sólo Argentina, sino también países como Cuba, Brasil o México, aumentaron su participación como socios comerciales de España. Fue la etapa hispanoamericana, buscada por el gobierno de Franco más por necesidad que por vocación.

A partir de 1951 las importaciones españolas experimentaron, en términos reales, un notable crecimiento. La importación española creció a una tasa anual del 20 por 100 entre 1950 y $1958^{4}$. La importancia de las compras a los países de Iberoamérica disminuyó rápidamente a partir del cambio de decenio, a la vez que los países europeos recuperaban su papel protagonista como proveedores de la economía española. Como se verá más adelante, el cambio fue también evidente en la composición por mercancías de las importaciones. En cuanto a la exportación, las ventas de productos españoles crecieron de forma continuada aunque lenta entre 1946 y 1953, pasando de los 178 millones de dólares del primer año de posguerra (1.946 millones de pesetas), a los 481 de media en el primer lustro de los cincuenta (aproximadamente 13.000 millones) ${ }^{5}$. De este modo, los ingresos por exportación que en 1946-1947 significaban aproximadamente la mitad del total de ingresos totales, alcanzaron una participación de más del 70 por 100 en el bienio 1951-1952. Este aumento bastó para mantener el pulso a la importación durante el primer tercio de los años cincuenta. La tasa de cobertura se mantuvo entre 70 y 80 por 100 desde 1947 hasta 1953, reduciéndose a partir de ese año paulatinamente hasta alcanzar el mínimo 46,58 por 100 de 1956 (Gráfico 1).

También en la balanza de servicios se registraron algunos cambios significativos a partir de 1950. El superávit conseguido en los años de la Segunda Guerra Mundial fue reduciéndose progresivamente una vez aca-

4 Los cálculos están basados en los datos de Martínez Ruiz (1998) convertidos a pesetas a los cambios medios ponderados calculados por Asensio (1995).

s Con la excepción del año 1950 en que la exportación cayó. Al contrario que en el caso de la importación, los nuevos datos de exportación ofrecen un perfil muy diferente del que era habitual hasta ahora. Véase Martínez Ruiz (1998). Nótese que, en ocasiones, la conversión a pesetas de las cifras originales en dólares tiene como resultado variaciones significativas en algunas series y magnitudes tales como el saldo comercial, la tasa de cobertura o la evolución de la balanza básica. 


\section{GRÁFICO 1 \\ Evolución del comercio español}

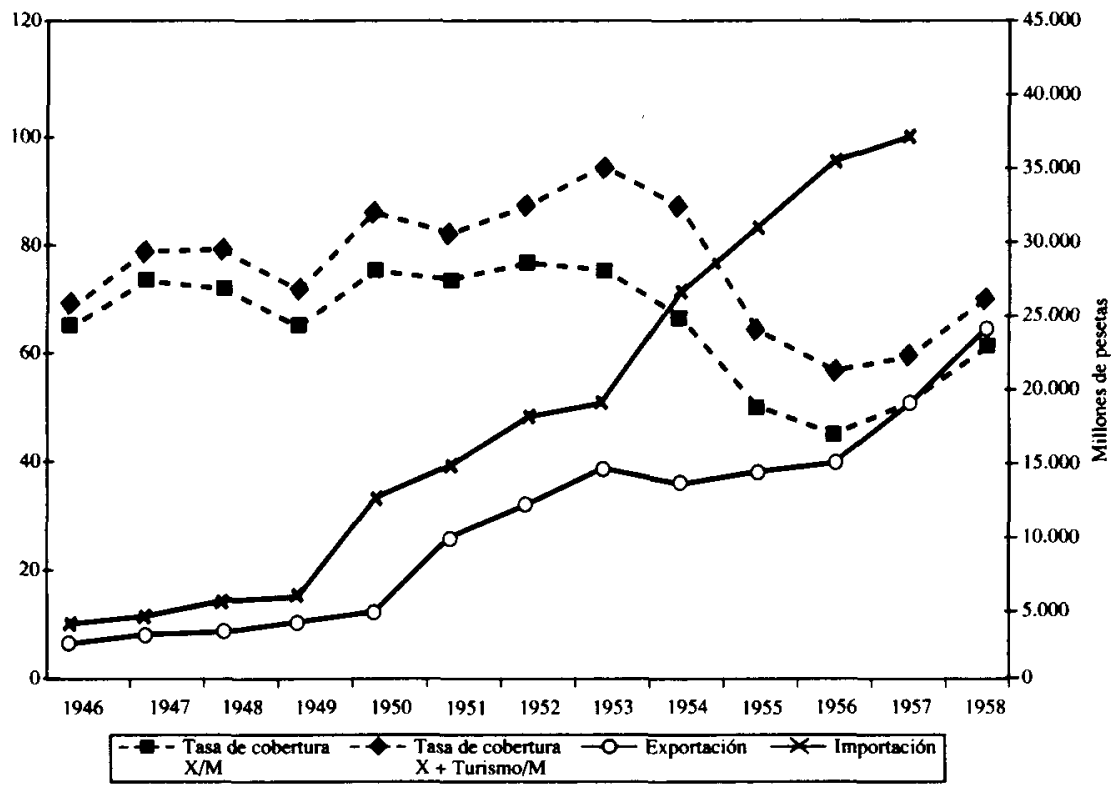

FuENTES: Martínez Ruiz (1998). Tipos de cambio medios ponderados en Asensio (1995).

bado el conflicto, hasta convertirse en déficit en 1947. Las razones fueron varias. En primer lugar, cayeron de manera continuada los ingresos obtenidos por servicios de fletes, a la vez que paralelamente aumentaron las salidas por ese concepto. También se produjo un aumento en los pagos en concepto de rentas de capital, causado por las obligaciones contraídas con la República Argentina. A partir de 1951, sin embargo, el crecimiento de los ingresos llevó a la balanza de servicios a un creciente superávit, que pasó de los 5 millones de dólares (241 millones de pesetas) de 1952 a los más de 105 millones de 1954 (4.271 millones de pesetas). En esos años se registró una mejoría en casi todos los conceptos que integran la balanza de servicios, sin embargo, el gran protagonista fue el turismo. Los ingresos obtenidos por las visitas de ciudadanos extranjeros aumentaron notablemente a partir de 1949, año en el que se registró la entrada de 284.000 viajeros que consumieron bienes y servicios por un valor apro- 
ximado de 350 millones de pesetas. En 1954, más de 1,3 millones de turistas permitieron a la economía española ingresar más de 4.300 millones de pesetas. Unidas a la exportación, estas entradas llegaron a financiar más del 95 por 100 de la importación española (Gráfico 1). En otro orden de cosas, el saldo de la balanza de transferencias que había arrojado un saldo muy positivo, desde el final de la Guerra Mundial, sufrió una drástica reducción a partir de 1950, una vez se consumó la ruptura con Argentina. Las remesas procedentes de Argentina habían sido las grandes protagonistas. Entre 1946 y 1950 más de 26,5 millones de dólares fueron enviados desde el país sudamericano como auxilio familiar, lo que suponía más del 55 por 100 del total de las transferencias de familiares recibidas desde el exterior. Este volumen de ingresos no volvió a alcanzarse hasta después de 1953, gracias, esta vez, a las donaciones norteamericanas. En resumen, entre 1950 y 1954 los ingresos por invisibles se multiplicaron por más de siete, pasando de poco más de 1.300 millones de pesetas anuales en 1950 a 8.100 millones en 1954, lo que resultó fundamental para que el déficit de la balanza por cuenta corriente se mantuviese controlado.

El inicio del decenio de los cincuenta coincidió con un significativo cambio en los movimientos registrados en la balanza de capital. Entre 1946 y 1949, las cuantiosas sumas ingresadas en forma de préstamos permitieron financiar los déficit de la balanza por cuenta corriente. En estos años se recibió un total de 352,26 millones de dólares en concepto de préstamos intergubernamentales a largo plazo, créditos que procedían en su mayor parte de Argentina. Los créditos argentinos fueron la única válvula de escape de la economía española. El saldo de la balanza de capital, que había registrado grandes superávit hasta 1949, apenas superó los 10 millones de dólares entre 1950 y 1952 y fue negativo en el bienio 1953-1954. En general, durante el período autárquico, las autoridades españolas renunciaron a la inversión externa directa como consecuencia de su ideología nacionalista, rechazo que queda reflejado en la ausencia total de entradas registradas como inversión directa en empresas hasta 1950 y su lentísimo crecimiento a partir de ese momento. A pesar de ello, la entradas de capital privado supusieron más de un 90 por 100 de las entradas totales de capital registradas entre 1950 y $1958^{6}$. Entre éstas se pueden destacar las entradas registradas en las cuentas oficiales como «importación definitiva de capital», rúbrica que pretendía recoger la repatriación del capital español salido al

- Con la excepción del año 1956 en que el gobierno español recibió préstamos del gobierno de EE UU en el marco de los acuerdos firmados en 1953. 
extranjero durante los años veinte y treinta, y que comienzan a ser de importancia en 1953, año en que, por primera vez, se superaron los 300 millones de pesetas ${ }^{7}$. A partir de ese año y hasta 1958, la repatriación de capitales supuso más de 5.800 millones de pesetas. Otras entradas de capital privado de cierta importancia fueron las obtenidas mediante el crédito concedido por el Export-Import Bank a diferentes empresas españolas, que comenzó a utilizarse a partir de 1951 y supuso casi 4.000 millones de pesetas hasta 1958. La llegada de los fondos otorgados en préstamo por los bancos privados norteamericanos sólo consiguió sustituir de manera parcial a los flujos argentinos a partir de 1949. El volumen de estos créditos fue mucho menor que el de los argentinos y las condiciones de pago mucho más onerosas para la economía española, lo que a la postre llevó a su cancelación ${ }^{8}$.

Un indicador de la integración de la economía española en los mercados internacionales, como es la tasa de absorción, confirma la escasa conexión con los mercados financieros mundiales durante los años cincuenta. Así, los resultados correspondientes a la segunda mitad de los cuarenta, arrojan una media por encima del 0,8 , frente al escaso 0,35 obtenido para los años iniciales de la década de los cincuenta, lo que pone de manifiesto la mayor significación de los ingresos registrados en la balanza de capital entre 1946 y 1949. Puede concluirse, por tanto, que la importación de capital no cobró protagonismo hasta los años sesenta. Esto supuso una limitación del potencial de crecimiento de la economía española al supeditar la inversión a la capacidad de ahorro interno y la compra de materias primas, bienes intermedios $y$, sobre todo, maquinaria y equipos, a la capacidad de exportación de productos y servicios españoles.

\section{DIVISAS Y CRECIMIENTO}

A pesar de la renuncia al capital extranjero, el incremento de los ingresos de divisas fue muy notable a partir de 1951. Las entradas totales de divisas se dispararon a partir de 1950, pasando de un nivel aproximado de 7.000 millones de pesetas a un máximo de 42.000 millones en 1958. Incluso

${ }^{7}$ Estas entradas pueden tener relación con el favorable marco que significó el programa dólar-peseta, aunque quizá la confianza que generó la firma de los acuerdos hispano-estadounidenses influyera en esta repatriación de capital. Sobre los efectos del tratado sobre la economía española, véase Calvo González en este mismo número de la Revista de Historia Económica (pp. 253.275).

${ }^{*}$ Martínez Ruiz (1998). 
en términos relativos la creciente significación de las entradas de divisas es apreciable. La cuota de ingresos exteriores sobre PIB pasó de alrededor del 4 por 100 en la década de los cuarenta, al 6 y, a partir de 1953, al 8 por 100 , todo ello a pesar del notable crecimiento del producto español en los años cincuenta 9 . La mayor disponibilidad de divisas hizo posible el aumento de las importaciones. Hay que resaltar el progresivo aumento de las compras de primeras materias registrada en estos años, sobre todo de las no energéticas, que vino a paliar la escasez de inputs productivos en que se había desarrollado la producción hasta 1949. En conjunto, la importación de materias primas y bienes intermedios pasó de una media anual de 170 millones de dólares en la segunda mitad de los cuarenta, a 240 millones entre 1951 y 1955 . Sin embargo, la importancia relativa de este grupo de bienes al que durante todo el período autárquico correspondió la parte del león de las compras exteriores, no aumentó, manteniéndose entre el 50 y el 60 por 100 de las importaciones totales. El mayor aumento relativo se registró en el capítulo de bienes de capital. Entre 1949 y 1954, las importaciones de maquinaria aumentaron hasta constituir aproximadamente el 25 por 100 de las compras totales, cifra que hasta ese momento apenas había superado el 11 por 100 . Si la restringida capacidad para importar constituyó el principal obstáculo al crecimiento económico durante los años cuarenta, el aumento de las compras exteriores a partir de 1950 significó la relajación de esa limitación, favoreciendo el despegue de la economía española, que contó, a partir de 1950, con una mayor cantidad y variedad de insumos.

Entre los más importantes, sin duda, destacan la mayor disponibilidad de maquinaria y equipos. La revitalización de las compras de equipo y maquinaria fue sin duda especialmente beneficiosa para el crecimiento de la economía española. Como han sugerido varios autores, las importaciones de bienes de capital no sólo suponen una transferencia tecnológica para una economía, sino que además contribuyen a una mayor eficiencia en el proceso de acumulación, debido al menor precio relativo de los bienes de equipo en los países más desarrollados ${ }^{10}$. La significación de los bienes de capital extranjero en el conjunto de la inversión aumentó en España a partir de 1949. Como puede verse en el Gráfico 2, durante la primera mitad de los cincuenta, una parte cada vez mayor de la creciente inversión española se destinó a equipos y maquinaria de procedencia extranjera. La

" Cálculos basados en los datos ofrecidos en Martínez Ruiz (1998) y Prados de la Escosura (1995).

${ }^{10}$ Lee (1994). 


\section{GRÁFICO 2}

\section{Cuota de inversión dedicada a bienes importados}

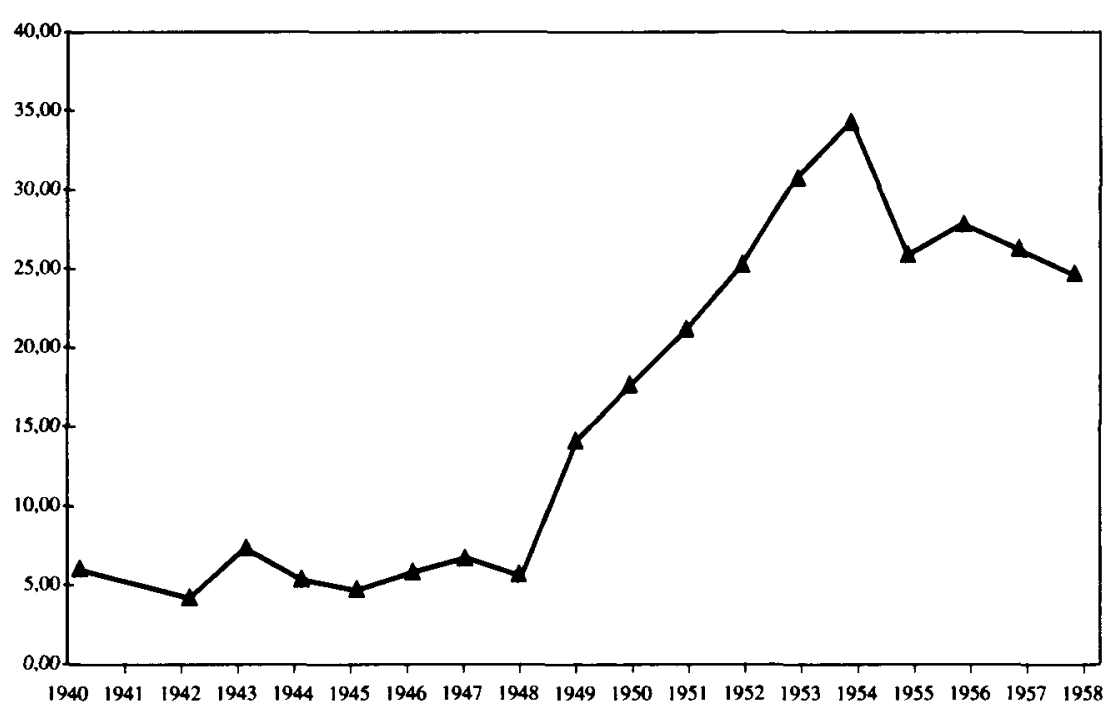

Nota: La cuota de importaciones en la inversión $(Z)$ ha sido calculada según la fórmula $Z=I_{M} / I_{D}$, donde $I_{M}=$ Importación de bienes de capital $e I_{D}=$ Producción interna de bienes de capital. Véase Lee (1994).

Fuentes: Los datos de inversión en maquinaria y equipo en Prados de la Escosura (2001), la importación de bienes de capital ha sido calculada a partir de los datos de comercio proporcionados por la Dirección General de Aduanas, mientras la producción interna de bienes de capital es la diferencia entre las variables anteriores.

creciente participación de los bienes de capital importados en la inversión podría haber contribuido a acelerar el ritmo de crecimiento de la economía española durante los años cincuenta, pues supuso la incorporación al proceso productivo de tecnologías más eficientes y ayudó a reducir la pérdida de eficiencia en la actividad inversora que suponía la opción de desarrollo autárquica.

Por tanto, el aumento de las entradas de divisas relajó especialmente la restricción que pesaba sobre las compras de maquinaria. Esta restricción puede explicarse atendiendo a las prioridades mostradas por las autoridades comerciales a la hora de conceder las licencias de importación. Los productos alimenticios, por un lado, y los carburantes y materias primas impres- 
cindibles para garantizar el funcionamiento de la actividad productiva, por otro, gozaron en todo momento de un tratamiento preferente, mientras las importaciones de maquinaria y de aquellos bienes considerados de lujo quedaban postergadas. En este caso, el incremento en las compras exteriores de maquinaria podría venir simplemente dado por un aumento de los ingresos de divisas del país. Es decir, la importación de bienes de equipo no habría estado determinada por la demanda, sino por la oferta, que a su vez dependía del volumen de medios de pago disponibles ${ }^{11}$. En efecto, las preferencias reveladas por las autoridades comerciales españolas confirman que los criterios de asignación de divisas vigentes durante la autarquía respondían a un esquema de este tipo, donde las importaciones de maquinaria ocupaban el último lugar en la lista de prioridades. Por ejemplo, el orden de reparto de los medios de pago anteponía las peticiones de los ministerios y organismos oficiales a las solicitudes tramitadas a través de la dirección general de Comercio. Las peticiones oficiales incluían las importaciones de una larga lista de alimentos y productos energéticos, centralizadas en la Comisaría General de Abastecimientos y Transportes y el Ministerio de Hacienda respectivamente, además de las atenciones de gastos corrientes y obligaciones financieras generales. Una vez satisfechas estas necesidades, las restantes divisas pasaban a formar parte del llamado «cupo de Industria», que constituía el volumen total de divisas al que de hecho tenían acceso los agentes privados ${ }^{12}$. Teniendo en cuenta que una cantidad dada de materias primas era imprescindible para mantener viva la actividad productiva, fácilmente se puede deducir que el montante de divisas dedicado a la importación de bienes de equipo quedaba reducido al resto, fuese éste mayor o menor. Algunos datos corroboran este extremo. Así, por ejemplo, a pesar del crecimiento de las importaciones de bienes de consumo y alimentos, la cuota de divisas dedicadas a estas compras, que alcanzó aproximadamente el 35 por 100 de media anual entre 1946 y 1950, se redujo a partir de ese momento hasta el 15 por 100 . Por el contrario, entre 1951 y 1958, las divisas cedidas para la importación de maquinaria

${ }^{11}$ Esta posibilidad fue apuntada por Díaz Alejandro (1970) para Argentina.

12 Estos aspectos son tratados con más detalle en Martínez Ruiz (2000). Un caso singular lo constituye el Instituto Nacional de Industria. En principio, las importaciones solicitadas por las empresas del INI pasaban al régimen general de autorización, es decir, se cargaban en el llamado «cupo de Industria». Dentro de este cupo, sin embargo, gozaban de prioridad frente a las peticiones de los agentes privados (O. de 24 de marzo de 1944). Sin embargo, es muy probable que los máximos responsables del INI hicieran valer sus relaciones y posición dentro de la Administración española, para conseguir divisas adicionales para sus compras. 
y equipos significaron entre el 24 y el 34 por 100 del total, cifra que en el decenio de los cuarenta apenas había superado el 15 por 100.

\section{LA POLÍTICA COMERCIAL EN EL CAMBIO DE DECENIO}

La política económica exterior de los primeros veinte años del régimen franquista se caracterizó por la amplitud y la intensidad de la intervención del Estado, articulada a través de un exhaustivo sistema de control de cambio fuertemente restrictivo, definido por la total inconvertibilidad de la peseta, tanto interna como externa, el monopolio absoluto del Estado en cuestión de comercio y tenencia de divisas y la constante sobrevaloración de la valuta ${ }^{13}$. Precisamente para contrarrestar los perniciosos efectos de la política cambiaria se acudió a la política comercial, aunque, como es sabido, se despreciaron casi por completo los instrumentos tradicionales, como barreras arancelarias, sustituyéndose por una compleja red de restricciones cuantitativas y acuerdos bilaterales. Las relaciones exteriores estuvieron, por tanto, rígidamente reglamentadas en un régimen de autorización previa que abarcaba tanto el comercio como las operaciones financieras de todo tipo.

En el marco de este sistema se fueron introduciendo algunas modificaciones significativas, entre las que destaca la creación del sistema de cambios múltiples en diciembre de 1948. Los cambios múltiples o especiales no eran sino un sistema de subvenciones y tasas, por el que se pretendía fomentar la exportación y las operaciones de invisibles y desincentivar la importación. También el régimen de cambios especiales fue objeto de algunas reformas durante los diez años de su existencia. La más importante de ellas fue el paso de un sistema de tipos múltiples fijos a uno de tipos múltiples flexibles en 1950. Este paso se verificó con la creación de un mercado de divisas, fuertemente controlado a pesar de ser llamado «libre». A partir de 1950, los tipos de cambios no fueron fijados exclusivamente por las autoridades monetarias y comerciales, sino que eran el resultado de una combinación variable del tipo de cambio oficial y del vigente en el mercado, lo que suponía una novedad en el sistema español de control de cambios. ¿Constituyeron estas reformas el inicio de nuevo camino «liberalizador»? ¿Cómo influyeron en la evolución de las relaciones económicas internacionales?

${ }^{13}$ A este respecto puede consultarse Eguidazu (1978), Viñas et al. (1979) o Martínez Ruiz (2000). 
Para responder a estas cuestiones es preciso analizar las circunstancias que llevaron a la introducción de los tipos de cambios múltiples. La implantación de los cambios especiales vino precedida, como se vio anteriormente, de tres años de grandes dificultades en la balanza por cuenta corriente. El fracaso de la política económica exterior de los primeros gobiernos franquistas se puso claramente de manifiesto una vez acabada la Segunda Guerra Mundial. A pesar de las entradas de capital, la falta de divisa libres, sobre todo dólares, y un supersticioso rechazo al endeudamiento exterior hicieron que las autoridades españolas mostraran una creciente preocupación ante la evolución del sector exterior. La solución finalmente elegida por las autoridades fue la aplicación de un alambicado sistema de tipos de cambio múltiples. Sin embargo, esta decisión se retrasó más de año a partir de que los responsables del IEME llamaran la atención del ministro sobre la grave situación del sector exterior ${ }^{14}$. Probablemente la razón de este retraso haya que buscarla en las expectativas de encontrar ayuda financiera exterior. En junio de 1947, se había anunciado un nuevo programa de ayuda a la reconstrucción europea, el Programa de Recuperación Europea, luego conocido como Plan Marshall. A este plan de ayudas podrían acogerse todas las naciones europeas, con la condición de que éstas se uniesen en una organización que asegurase la utilización eficiente de los fondos. España intentó en vano ser incluida en tal programa ${ }^{15}$. Las autoridades españolas esperaban del plan Marshall beneficios políticos y económicos. En lo político hay que destacar, no sólo la ruptura del bloqueo decretado contra España en 1945, sino sobre todo, la homologación del régimen con los países de Europa Occidental. En cuanto a los aspectos económicos, además de la posibilidad de aumentar las importaciones de alimentos, materias primas y bienes de equipo, la inclusión en el Plan Marshall habría hecho posible el acceso a créditos en dólares, hasta entonces vedados, y la normalización del comercio con los países europeos, así como la reanudación de las relaciones económicas con Alemania.

No obstante, todas las gestiones españolas frente a las autoridades norteamericanas resultaron infructuosas. El principal obstáculo fue la reticencia de los restantes países europeos a colaborar políticamente con el régimen franquista, reticencia que ni las misiones diplomáticas españolas ni el apoyo de Salazar pudieron vencer ${ }^{16}$. En abril de 1948, conversaciones directas

14 Martínez Ruiz (2000).

15 Abundante información sobre los intentos españoles para entrar en el Plan Marshall se encontrará en Viñas y otros (1979), pp. 474 ss., así como en Guirao (1998), pp. 86 ss.

16 Guirao afirma que tampoco el gobierno estadounidense era partidario de incluir a España en el Plan. Guirao (1998), pp. 57.61. 
con dirigentes norteamericanos, así como diversas manifestaciones del presidente Truman dejaron claro que EE UU no iba a oponerse a la opinión de sus aliados europeos en este asunto, aunque se dejaba la puerta abierta al estrechamiento futuro de las relaciones bilaterales. Una vez frustradas las primeras expectativas de entrar en el Plan Marshall, el gobierno español hubo de enfrentarse a los problemas causados por la escasez de divisas y lo hizo intensificando la intervención en el sector exterior, con la introducción de un nuevo instrumento de control, los tipos de cambio múltiples ${ }^{17}$. De este modo, el régimen español pasó a ser un sistema compuesto, donde se combinaban restricciones cuantitativas y de coste, y, además, incluía un mercado parcialmente libre en el que se autorizaba la negociación de determinadas divisas destinadas a operaciones concretas. La moneda extranjera era vendida en ocasiones a tipos de cambio que incorporaban una penalización, cuyos ingresos eran destinados, al menos parcialmente, a subvencionar algunas exportaciones o importaciones. Según describe el FMI, estas características eran las que definían el «modelo latinoamericano» de restricciones cambiarias ${ }^{18}$. $\mathrm{Si}$, como mantuvieron $\mathrm{De}$ Long y Eichengreen, la llegada del Plan Marshall significó en Europa occidental el desarrollo de instituciones y políticas proclives al mercado libre, la exclusión del Plan empujó a las autoridades españolas hacia una intensificación del intervencionismo, al menos en el ámbito de las relaciones exteriores, lo que alejó a España de la senda de liberalización emprendida por otros países europeos, como Francia o Italia ${ }^{19}$.

Hay que resaltar que, a pesar de las llamativas novedades, las características generales del sistema no cambiaron hasta el Plan de Estabilización, porque las licencias continuaron constituyendo el verdadero mecanismo limitador de la demanda de medios de pago, aun después de la introducción de mecanismos de coste tan poderosos como los cambios múltiples. La cuota de licencias denegadas se mantuvo entre un 75 y un 85 por 100 entre 1951 y 1958 , lo que puede ser tomado como un indicador del gran volumen de demanda que fue eliminado gracias al sistema de autorización

${ }^{17}$ En los meses que median, un crédito concedido por la Banca Nacional Suiza vino a aliviar la situación.

${ }^{18}$ FMI (1950), pp. 14-15.

${ }^{19}$ De Long y Eichengreen (1993). Prados de la Escosura y Sanz (1996), p. 363, proponen precisamente la utilidad del caso español como contrafactual de lo que habría sucedido en Europa sin el Plan Marshall. El caso del control de cambios sería un buen ejemplo del desarrollo sin acceso al programa de ayudas. 
previa ${ }^{20}$. La introducción de los cambios múltiples, por tanto, no puede ser considerado un síntoma de apertura, porque las restricciones cuantitativas no se relajaron, perdiéndose así la oportunidad de introducir el precio de la divisa como mecanismo de asignación de los medios de pago.

Esta afirmación puede aplicarse también al cambio de ministro. La alusión al «cambio de talante» que se habría hecho patente en la política comercial española a partir de 1951 es una constante en la literatura sobre el período ${ }^{21}$. La llegada de Arburúa al Ministerio de Comercio en verano de 1951 coincidió, según esta extendida opinión, con un cambio de rumbo en dirección a una política comercial y cambiaria más racional. La aparición del mercado libre, unida a la reducción del número de grupos de mercancías decidida en octubre de 1950 y la relajación en la concesión de licencias de importación son señaladas como las principales muestras de las intenciones del nuevo responsable de Comercio. Sin embargo, hay que destacar que las reformas más importantes fueron decididas con anterioridad y se deben en su totalidad a Suanzes ${ }^{22}$. La creación de un mercado, donde los agentes pudieran negociar una parte de sus divisas, estuvo inspirado en el modelo italiano y venía siendo estudiado desde 1948. Del mismo modo, la reducción del número de grupos dentro del sistema de cambios múltiples se comenzó a plantear a finales de 1949, después de que se pusiera de manifiesto que la excesiva complejidad y arbitrariedad del sistema lo hacían prácticamente inmanejable. A su llegada al sillón ministerial, Arburúa se encontró con las reformas, decididas y puestas en marcha por el anterior equipo, por lo que difícilmente puede considerarse que fueran muestra de su distinta concepción de la política comercial o cambiaria.

Por otro lado, no hay ninguna prueba de una mayor liberalidad en la concesión de licencias. Cierto es que, de acuerdo a los escasos datos

${ }^{20}$ Estas cifras reflejan la avalancha de peticiones de importación que se registró en las oficinas del Ministerio de Comercio durante estos años. En general, puede afirmarse que esta afluencia fue debida a la gran demanda de importación, muy superior a las posibilidades de financiación de la economía española, y que iba en aumento a causa del crecimiento económico. Sin embargo, también es necesario señalar que, en parte, el gran número de solicitudes se explica por la adaptación de los agentes a la arbitrariedad y falta de criterios públicamente conocidos en la concesión de licencias que hacían racional cursar un gran número de solicitudes para ver autorizadas alguna de ellas. Otro aspecto destacable es el aumento de la importancia de las importaciones realizadas fuera del sistema general de autorización que fue debido, de un lado, al incremento de las compras centralizadas por el Estado y a las importaciones realizadas en el marco de los convenios hispano-estadounidenses y, por otro, al contrabando recogido en las cifras que aquí se presentan. Martínez Ruiz (1998).

${ }^{21}$ Véase, por ejemplo, Ros Hombravella et al. (1973), Viñas et al. (1979), Asensio (1995).

22 Martínez Ruiz (2000), pp. 200-201. 
disponibles, entre 1951 y 1952 tanto el número como el valor de las licencias concedidas aumentaron notablemente y, además, se mantuvieron en las mismas magnitudes hasta el final del decenio ${ }^{23}$. Sin embargo, es necesario señalar que carecemos de indicación alguna sobre los datos referidos a los años inmediatamente anteriores, lo que inevitablemente disminuye la capacidad ilustrativa de este aumento. Bien podría suceder que el año 1951 fuese un año extraordinariamente negativo en cuanto a la concesión de licencias. Así parece indicarlo el hecho de que los datos acerca del número de licencias concedidas entre 1943 y 1946 reflejen unos valores semejantes, e incluso muy superiores, a los de $1951^{24}$. La caída de la exportación en 1950 o la interrupción de la normal actividad del ministerio debida a los cambios en el equipo dirigente, cuyos máximos responsables, recordemos, debían decidir sobre cada licencia, podrían aportar una explicación a esta supuesta moderación de las cifras de 1951. No quiere afirmarse aquí que fuese esto lo que ocurrió, sino tan sólo argumentar que los datos disponibles no permiten hablar de una mayor liberalidad en el régimen de autorización previa de las importaciones.

En cualquier caso, y aunque no se encuentren pruebas de la voluntad de cambio de rumbo, algunos aspectos del nuevo régimen de política comercial fueron beneficiosos para la economía española. En primer lugar, los cambios múltiples incluyeron una fuerte devaluación de la peseta, que se acentuó en cada modificación o simplificación del sistema de cambios especiales para compensar en parte las diferencias en la evolución de los precios entre España y el exterior. La primera causa del aumento de la exportación española a partir de 1949 podría ser precisamente la devaluación de la peseta decidida implícitamente con la introducción del régimen de cambios múltiples. Así lo indican Serrano Sanz y Asensio cuando afirman que la devaluación posiblemente «tuviera consecuencias decisivas en la mayor holgura de la balanza de pagos durante la primera mitad de los cincuenta» ${ }^{25}$. Según los cálculos de estos autores, el tipo de cambio efectivo real para el comercio sufrió una drástica devaluación entre 1948 y 1951. Esta caída

${ }^{23}$ El primero pasó de 10.445 a 20.149 , mientras el valor en dólares aumentó desde los 248 millones de 1951 a los 395 de 1952.

${ }^{24}$ El número de licencias concedidas varió enormemente en estos años de guerra. Así, mientras en 1943 se concedieron 40.266 licencias de las 45.960 solicitadas, un extraordinario 87,61 por 100 , en 1944 y 1945 el número de licencias aprobadas osciló entre las 15.000 y las 19.000. En 1946, esta cifra volvió a aumentar hasta más de 30.000. Martínez Ruiz (2000), pp. 168 y 188.

${ }_{25}$ Serrano Sanz y Asensio (1997), pp. 560-561. Para la estimación de la sobrevaloración de la peseta, pp. 566-569. 
del valor de la peseta hubo de favorecer, sin duda, las ventas de productos españoles en el exterior.

La corrección de la sobrevaloración de la peseta a través del llamado Programa dólar-peseta pudo tener también alguna influencia en el aumento de los ingresos de invisibles, si bien algo más tarde, pues se puso en marcha en 1952. El programa se articulaba a través de un sistema semejante a un acuerdo de compensación privada, donde los importadores españoles pagaban sus compras en pesetas al IEME, mientras la WCC se ocupa de pagar a los exportadores norteamericanos en dólares. Estos dólares a su vez procedían bien de turistas extranjeros con destino a España, bien de las aportaciones de agentes privados que deseaban hacer llegar a España pagos en concepto de rentas financieras, auxilio familiar o repatriaciones de capital. El incentivo para utilizar este mecanismo venía dado por el favorable tipo de cambio que el IEME concedía a las operaciones negociadas a través de la WCC, que acercaba el cambio oficial al vigente en el mercado de Tánger, lo que permitió detraer a este mercado buena parte de la demanda de pesetas durante algunos años ${ }^{26}$. Aunque este acuerdo tenía como principal objetivo facilitar a la industria textil catalana la importación de algodón en rama, es muy probable que durante un tiempo tuviera un efecto positivo sobre los ingresos de divisas por invisibles ${ }^{27}$.

\section{LA INFLUENCIA DEL CONTEXTO INTERNACIONAL}

Resulta en todo caso muy difícil dilucidar hasta qué punto la devaluación afectó a las exportaciones españolas, porque el cambiante contexto internacional, tanto en lo que respecta al plano económico como en el político, trajo consigo una considerable modificación del escenario en el que discurría el comercio español. Para comprobar la importancia de cada factor en el crecimiento de la exportación se ha realizado un análisis de «cuotas de mercado constantes», para el decenio de los cincuenta, dividido en dos lustros ${ }^{28}$. El Cuadro 1 recoge los resultados. De acuerdo con éstos, los buenos resultados del sector exportador entre 1949 y 1953 se apoyaron, en primer lugar, en el auge de la economía mundial que comenzaba a

${ }^{26}$ Esta operación se analiza más detalladamente en Martínez Ruiz (2000). El programa mostró sus límites a partir de 1955 , cuando el aumento de la presión inflacionista hizo que la cotización de la peseta en Tánger volviera a bajar.

${ }_{27}$ El fin último del Programa era minimizar la diferencia entre el tipo de cambio de Tánger y el oficial.

${ }_{28}$ Sobre los límites de este tipo de análisis véase Richardson (1971). 


\section{CUADRO 1}

Análisis de cuotas de mercado constante para la exportación española

\begin{tabular}{lrcc}
\hline & $\begin{array}{c}\text { Efecto } \\
\text { demanda }\end{array}$ & $\begin{array}{c}\text { Efecto } \\
\text { competitividad }\end{array}$ & $\begin{array}{c}\text { Efecto } \\
\text { integración }\end{array}$ \\
\hline $1949-1953 \ldots \ldots \ldots . .$. & 49,10 & 34,18 & 16,72 \\
$1954-1958 \ldots \ldots \ldots . .$. & 131,67 & $-25,53$ & $-6,14$ \\
$1949-1958 \ldots \ldots \ldots . .$. & 79,04 & 10,77 & 10,18 \\
\hline
\end{tabular}

FueNTES: United Nations (1962) y Martínez Ruiz (1998).

vivir su etapa dorada de crecimiento. La pujante demanda internacional, ayudada por el boom causado por la Guerra de Corea, tiró de los valores de la exportación española. No obstante, también se registró un importante aumento de la competitividad que puede explicar casi el 35 por 100 del crecimiento de la exportación española en estos años. Las cifras son menos optimistas cuando se refieren al decenio en su conjunto. El dinamismo de la exportación española se frenó a partir de 1953, a pesar del notable aumento del comercio internacional, y fue perdiendo competitividad entre 1954 y 1958, todo ello a pesar de la devaluación de 1957. Como sugirió Tena, el favorable contexto internacional fue aprovechado en menor medida por España que por sus más directos competidores, lo que debe achacarse a la todavía notable sobrevaloración de la peseta y la intensidad del aparato protector en vigor ${ }^{29}$. La escasa modernización del sector productivo español, veinte años después del final de la Guerra Civil, se refleja de forma clara en la composición de la exportación. A finales del período considerado, los principales productos de exportación continuaban siendo los agrios, el mineral de hierro y los aceites, tanto de oliva como minerales. Los alimentos constituyeron más del 50 por 100 de la exportación española durante toda la década de los cincuenta, mientras la cuota de las materias primas en la exportación oscilaba entre el 20 y el 30 por 100. En un contexto internacional de creciente demanda de productos industriales, los productos manufacturados españoles apenas consiguieron elevar su participación en la exportación española, a pesar del notable crecimiento del

\footnotetext{
${ }^{29}$ Tena (1992).
} 
producto industrial dentro del país ${ }^{30}$. Los bienes de equipo tan sólo significaban el 1 por 100 de las ventas anuales al exterior.

Un elemento esencial en la explicación del aumento de las exportaciones españolas a partir de 1949 es el definitivo levantamiento del bloqueo internacional, una vez se impuso la doctrina Truman. El final del bloqueo abrió las puertas de los mercados internacionales a los productos españoles y permitió recuperar algunos de los principales clientes tradicionales ${ }^{31}$. La trascendencia de este hecho queda clara cuando consideramos que, por ejemplo, la apertura de la frontera y la consiguiente negociación de un nuevo acuerdo de pagos con Francia en 1948 y la reanudación de las relaciones comerciales con Alemania en 1949 son por sí solas responsables de más de un 30 por 100 del crecimiento de la exportación entre 1948 y 1951 , cuota que se eleva hasta el 50 por 100 si la referimos a las entradas de divisas por venta de productos. La recuperación del comercio español con sus tradicionales socios europeos fue potenciada por la escasez de divisas fuertes, especialmente de dólares, que padecían la mayor parte de los países de Europa Occidental lo que forzó una desviación del comercio hacia aquellos países que permitían el pago en divisa nacional o, incluso, que comerciaban preferentemente en el marco de acuerdos de pago bilaterales o clearing. Éste era el caso de España. Entre 1948 y 1951, España negoció nuevos acuerdos de pagos con la mayor parte de las naciones europeas ${ }^{32}$. Gracias a estos tratados, España aseguró los mercados para sus exportaciones más tradicionales, tales como productos alimenticios y materias primas, e incluso consiguió imponer la compra de algunos productos adicionales. De esta manera, Europa Occidental volvió a constituirse como el principal destino de las exportaciones españolas.

La recuperación europea estuvo también en la raiz de los positivos resultados del turismo. El desarrollo de lo que más tarde se iba a convertir en la lucrativa industria turística española se inició en los primeros años del decenio de los cincuenta. Lejos de ser fruto de una acción planeada por los responsables de la política económica en España, quienes, sorprendidos por el creciente número de visitas de ciudadanos extranjeros, se vieron obligados a encargar planes de urgencia para satisfacer la creciente demanda a partir de $1950^{33}$, la explicación ha de buscarse en la bonanza económica que comenzaba a experimentarse en los países de Europa Occi-

\footnotetext{
10 Milward (1992).

3 Sobre el levantamiento del bloqueo, Portero (1989) y Guirao (1998).

32 Guirao (1998).

33 Archivo Banco de España, Extranjero, IEME, Secretaría, Cajas 2 y 6.
} 
dental. El fin del bloqueo internacional a partir del acercamiento de EE UU al gobierno de Franco abrió las fronteras españolas a un creciente número de franceses, británicos y alemanes, que pudieron disfrutar en España de su favorable situación económica ${ }^{34}$.

\section{CONCLUSIONES}

La escasez de divisas, que limitó la capacidad de importación, constituyó el principal cuello de botella a que se enfrentó la economía española durante el período autárquico. Sin embargo, esta limitación pareció relajarse, al menos parcialmente, a partir de 1950 , y durante el decenio siguiente las compras de bienes extranjeros crecieron a un ritmo superior al de la economía en su conjunto. En este trabajo se ha analizado, en primer lugar, la evolución de las principales partidas de la balanza de pagos española, constatándose que las entradas de divisas fueron fruto del incremento de la exportación, en especial entre 1950 y 1953 . Además, algunos capítulos de los servicios, principalmente el turismo, ayudaron a financiar la creciente importación. Por último, hay que señalar la escasa significación de las entradas de capital, públicas o privadas, en los años cincuenta, incluyendo en este capítulo la ayuda americana. La renuncia franquista a la financiación exterior se tradujo en un mínimo grado de integración de la economía española en los mercados internacionales de capital, decisión que supuso una rémora para el crecimiento económico a largo plazo.

En segundo lugar, se han analizado los factores que pudieron determinar el aumento de las entradas de divisas en la primera mitad del decenio de los cincuenta. La primera explicación estudiada es un cambio en la política económica. A pesar de que frecuentemente el cambio en la máxima responsabilidad del Ministerio de Comercio en 1951 es señalado como el inicio de una nueva etapa en la política comercial, ni la cronología de las reformas, ni los datos sobre concesión de licencias avalan esta interpretación. Tampoco la introducción de tipos de cambio múltiples significó una modificación real de la política comercial realizada hasta 1949, puesto que las licencias continuaron siendo el principal mecanismo regulador de las transacciones exteriores, perdiéndose así una excelente oportunidad para relajar el aparato proteccionista. Con todo, existieron algunos aspectos positivos. En primer lugar, la devaluación de la peseta implícita en los cambios

${ }^{34}$ Los porcentajes de ingresos por país de procedencia entre 1940 y 1952 en Martínez Ruiz (1998). 
múltiples colaboró en el aumento de la exportación a partir de 1951. En segundo lugar, el programa dólar-peseta sirvió para detraer al mercado negro parte de las divisas negociadas, lo que se reflejó en el aumento de las entradas por invisibles.

Sin embargo, a pesar de que puedan encontrarse ligeros rasgos favorables en la política económica, en general puede decirse que los buenos resultados del sector exterior tuvieron su origen en los cambios económicos y políticos en el mundo. En primer lugar, la recuperación económica europea y el boom de la Guerra de Corea impulsaron la demanda mundial, lo que benefició a la exportación española. En segundo lugar, la escasez de dólares y, en general, de divisas libres que sufría Europa Occidental forzó una orientación del comercio hacia países que admitían pagos en otras divisas o en el marco de acuerdos bilaterales de pagos, como era el caso de España. Por último, hay que mencionar el acceso a los mercados internacionales tras el levantamiento del bloqueo decretado por las Naciones Unidas, que hizo posible la recuperación de alguno de los más importantes clientes tradicionales, como Francia o Alemania. El favorable contexto internacional, tanto económico como político, dio un respiro a un sistema que a finales de los cuarenta estaba a punto de agotarse, permitiendo una revitalización de las relaciones económicas externas.

Durante un breve espacio de tiempo, el sector exterior dejó de ser un obstáculo insalvable para el crecimiento de la economía española. El aumento de los ingresos totales de medios de pago permitió, no sólo financiar la creciente corriente importadora, sino además modificar la composición de las compras exteriores. Así, la cuota de divisas dedicadas a la adquisición de maquinaria y equipos extranjeros aumentó a partir de 1950. Dado que las autoridades comerciales daban prioridad a otro tipo de importaciones a la hora de conceder licencias, las compras de maquinaria y equipos estuvieron en todo momento constreñidas por la oferta de medios de pagos. Sólo el incremento de los medios de pagos disponibles en el decenio de los cincuenta hizo posible el aumento de la importación de este tipo de bienes. Además, el crecimiento de la importación de bienes de equipo permitió aumentar la participación de la maquinaria extranjera en el conjunto de la inversión española a partir de 1949. Todo ello contribuyó a acelerar el crecimiento de la economía española, poniendo a disposición del sector productivo tecnologías más eficientes y haciendo más eficiente el proceso de acumulación. El dinamismo en las relaciones exteriores fue, sin embargo, efímero y, sobre todo, insuficiente, dado el grado de desabastecimiento de la economía española. 


\section{BIBLIOGRAFÍA}

Asensio, M. J. (1995): El proceso de apertura exterior de los cincuenta y el Arancel de 1960, Tesis doctoral, Universidad de Zaragoza.

De Long, J. B., y Eichengreen, B. (1993): «The Marshall Plan: History's Most Successful Structural Adjustment Program», en R. Dornbusch, W. Nolling y R. LAYARD (eds.), Postwar Economic Reconstruction and Lessons for the East Today, Cambridge Ma., MIT Press.

Diaz Alejandro, C. F. (1970): Essays on the Economic History of the Argentine Republic, New Haven-London, Yale University Press.

Dirección General de Aduanas (1940-1958): Estadistica del Comercio Especial, Madrid.

Eguidazu, F. (1978): Intervención monetaria y control de cambios en España, 1900-1977, Madrid, Editorial ICE.

GuIRAO, F. (1998): Spain and the Reconstruction of Western Europe, 1945-1957. Challenge and Response, Basingstoke-Hampshire-London, Macmillan Press Ltd.

InTERnATIONAl MONETARY Fund (1950): First Annual Report on Exchange Restrictions, Washington, IMF.

LEE, J. (1994): «Capital Goods Imports and Long-Run Growth», National Bureau of Economic Research, Working Paper, 4725.

Martín Aceña, P. (dir.); Martínez, E.; Martorell, M., y Moreno, B. (1998): Informe para la Comisión de Investigación de las transacciones de oro procedente del Tercer Reich durante la Segunda Guerra Mundial (Real Decreto 1131/1997, de 11 de julio), www.mae.es/holocausto (27 de noviembre de 2000).

Martínez Ruiz, E. (1998): «Las balanzas de pagos de la autarquía. Una revisión», Documento de trabajo 98-23, Universidad Carlos III de Madrid.

- (2000): El control de cambios en la España franquista: el Instituto Español de Moneda Extranjera, 1939-1973, Tesis doctoral, Universidad de Alcalá.

MILWARD, A. S. (1992): «El sector exterior en la expansión de los años cincuenta: comparación de las exportaciones españolas, italianas y portuguesas», en L. PrA. DOS DE LA ESCOSURA y V. ZAMAGNI (eds.), El desarrollo económico en la Europa del Sur: España e Italia en perspectiva bistórica, Madrid, Alianza, pp. 444-460.

Portero, F. (1989): Franco aislado. La cuestión española (1945-1950), Madrid, Aguilar.

Prados de LA Escosura, L. (1995a): «Spain's Gross Domestic Product, 1850-1993: Quantitative Conjectures», Working Papers, 95-05, Universidad Carlos III de Madrid.

- (1995b): «Spain's Gross Domestic Product, 1850-1993: Quantitative Conjectures. Appendix», Working Papers, 95-06, Universidad Carlos III de Madrid.

- (2001): Output and Expenditure in Spain 1850-2000 (en prensa).

Prados de la Escosura, L., y SANZ, J. C. (1996): «Growth and Macroeconomic Performance in Spain, 1939-93», en N. F. R. Crafts y G. Toniolo (eds.), Economic Growth in Europe since 1945, Cambridge, Cambridge University Press, pp. 355-387.

Richardson, J. D. (1971): «Constant-Market-Shares Analysis of Export Growth», Joumal of International Economics, 1, 2, pp. 227-239. 
Ros Hombravella, J.; Clavera, J.; Esteban, J. M.; Mones, M. A., y Montserrat, A. (1973): Capitalismo español: de la autarquía a la estabilización (1939-1959), Madrid, Cuadernos para el Diálogo.

Serrano Sanz, J. M., y Asensio, M. J. (1997): «El ingenierismo cambiario. La peseta en los años del cambio múltiple, 1948-1959», Revista de Historia Económica, 15, 3, pp. 545-573.

Tena Jungurto, A. (1992): «Protección y competitividad en España e Italia, 1890-1960», en L. Prados de la Escosura y V. Zamagni (eds.), El desarrollo económico en la Europa del Sur: España e Italia en perspectiva bistórica, Madrid, Alianza, pp. 321-358.

UnITEd NATIONS (1962): Yearbook of International Trade Statistics, New York.

Viñas, A.; Viñuela, J.; Eguidazu, F.; Pulgar, C. F., y Florensa, S. (1979): Política comercial exterior en España (1931-1975), Madrid, BEX. 